\title{
DeCÚBIto Ventral na SíndRome de Dificuldade RespiratóRIA no AdULto APÓS INFEÇÃO POR CORONAVÍRUS
}

\section{Posición PRONA EN LA SíNDROME DE DIFICULTAD RESPIRATORIA AGUDA DESPUÉS DE LA INFECCIÓN POR CORONAVIRUS}

\section{PRONE POSITION IN ACUTE RESPIRATORY DISTRESS SYNDROME AFTER CORONAVIRUS INFECTION}

DOI 10.33194/rper.2020.v3.s2.2.5777 | Submetido 25/05/2020 | Aprovado 11/11/2020

\author{
Mafalda Mota1 ${ }^{1}$; Luís Manuel Mota Sousa ${ }^{1,2}\left(\mathbb{D}\right.$; Isabel Bico' ${ }^{1}$; Maria do Céu Pinto Marques ${ }^{1,2}$ (D) \\ 1 - Universidade de Évora; 2 - Comprehensive Health Research Centre
}

\section{RESUMO}

Introdução: A nova infeção por corona vírus surgiu na China em dezembro de 2019, disseminou-se rapidamente pelo mundo e foi considerada pandemia pela Organização Mundial de Saúde em março de 2020. Esta infeção pode causar pneumonia com necessidade de internamento em unidades de cuidados intensivos. A posição de decúbito ventral é uma das intervenções que pode ajudar no tratamento do Síndrome de Dificuldade Respiratória Aguda destas pessoas.

Objetivo: Identificar os efeitos do decúbito ventral no tratamento de doentes com síndrome de dificuldade respiratória aguda no adulto causada por corona vírus.

Método: Revisão integrativa da literatura, realizada em maio de 2020. Foram utilizadas, para a colheita dos dados, as seguintes bases: SCOPUS, MEDLINE e Google Académico. Foi feita a aplicação dos critérios de inclusão e exclusão.

Resultados: Dos 241 artigos identificados, foram selecionados 13 artigos, todos publicados em 2020. Os dados foram sintetizados em 3 grandes temas, indicações, frequência da alternância de decúbito e duração e por último, os efeitos do decúbito ventral.

Conclusões: 0 decúbito ventral neste contexto, ajuda a melhorar os parâmetros ventilatórios e gasimétricos, os sintomas de dispneia, facilita a recuperação, com consequente, alta precoce, diminuição dos dias de internamento e redução da mortalidade aos 28 e 90 dias.

Palavras-chave: Decúbito Ventral; Infecções por Coronavírus; Síndrome do Desconforto Respiratório do Adulto; Enfermagem em reabilitação

\section{RESUMEN}

Introducción: la nueva infección por el coronavirus apareció en China en diciembre de 2019, se propagó rápidamente por todo el mundo y la Organización Mundial de la Salud la consideró una pandemia en marzo de 2020. Esta infección puede causar neumonía que requiere hospitalización en unidades de cuidados intensivos. La posición prono es una de las intervenciones que pueden ayudar en el tratamiento del síndrome de dificultad respiratoria aguda en estas personas.

Objetivo: identificar los efectos de la posición prona en el tratamiento de pacientes con síndrome de dificultad respiratoria aguda en el adulto causados por el corona virus.

Método: revisión integradora de la literatura, realizada en mayo de 2020. Las siguientes bases de datos se utilizaron para recopilar datos: SCOPUS, MEDLINE y Google Scholar. Se aplicaron los criterios de inclusión y exclusión.

Resultados: De los 241 artículos identificados, se seleccionaron 13 artículos, todos publicados en 2020. Los datos se sintetizaron en 3 temas principales, indicaciones, frecuencia de cambio de decúbito y duración, así como los efectos de la posición prono.

Conclusiones: La posición prono en este contexto, ayuda a mejorar los parámetros ventilatorios y gasométricos, los síntomas de disnea, facilita la recuperación, y consecuentemente, la alta temprana, la reducción de los días de hospitalización y la reducción de la mortalidad a los 28 y 90 días.

Palabras clave: Posición Prona; Infecciones por Coronavirus; Síndrome de Dificultad Respiratoria del Adulto; Rehabilitación de enfermería

\section{ABSTRACT}

Introduction: The new coronavirus infection appeared in China in December 2019, it spread rapidly throughout the world, and was considered a pandemic by the World Health Organization in March 2020. This infection can cause pneumonia requiring hospitalization in intensive care units. The prone position is one of the interventions that can help in the treatment of acute respiratory distress syndrome in these people. 
Objective: to identify the effects of the prone position in the treatment of patients with acute respiratory distress syndrome caused by the corona virus.

Method: integrative literature review conducted in May 2020. The following databases were used for data collection: SCOPUS, MEDLINE, and Google Scholar. Inclusion and exclusion criteria were applied.

Results: Of the 241 articles identified, 13 articles were selected, all published in 2020. The data was synthesized in 3 main subjects, indications, frequency of position changing and duration, and lastly, the effects of the prone position.

Conclusions: The prone position in this context, helps improve ventilatory and blood gas parameters, dyspnea symptoms, facilitates recovery, and consequently, early discharge, reduces hospitalization days and reduces mortality at 28 and 90 days.

Keywords: Prone Position; Coronavirus Infections; Respiratory Distress Syndrome, Adult; Rehabilitation nursing;

\section{INTRODUÇÃO}

Atualmente, o Ser Humano está a vivenciar uma fase particular da história. Sabe-se que a humanidade está rodeada de uma variedade de vírus já descobertos e ainda por descobrir. Enquanto que alguns vírus permanecem em hospedeiros específicos, existem outros que devido à sua rápida distribuição, afetam o ser humano, resultando em pandemias com elevada morbilidade e mortalidade; destas infeções, destacamse as infeções do sistema respiratório.

No último século, a humanidade deparou-se com grandes pandemias como a gripe por Influenzavirus A subtipo H1N1 em 1918 (gripe espanhola), a H1N1 influenza em 2009, a Severe Acute Respiratory Syndrome por coronavírus (SARS-CoV) em 2003 e a Middle East Respiratory Syndrome coronavirus (MERSCoV) em 2012. Estas pandemias apresentavam manifestações a nível respiratório, mas com grande risco cardiovascular e possível falência de órgãos ${ }^{(1)}$.

Atualmente há uma nova crise de saúde pública que ameaça o mundo, ou seja o surgimento e disseminação do novo coronavírus de 2019 (2019-nCoV) ou Severe Acute Respiratory Syndrome por coronavírus 2 (SARSCoV-2)(1-2). A Coronavirus Disease-2019 (COVID-19) é o resultado da SARS-Cov-2, causando síndrome de dificuldade respiratória ${ }^{(3)}$.

0 início da epidemia deu-se em Wuhan, China, em dezembro de 2019. As pessoas infetadas apresentavam sintomas relacionados com uma pneumonia, tendo sido posteriormente diagnosticados com o novo coronavírus (Covid-19). Devido à rápida disseminação do vírus, para outras regiões no país, mas também pelo mundo, incluindo 0 continente europeu, $\mathrm{o}$ continente americano, continente africano e na Oceânia, a Organização Mundial de Saúde, em março o presente ano, declarou a Covid-19 como uma pandemia ${ }^{(3)}$.

Primeiramente, a sintomatologia associada à infeção por SARS-CoV-2 inclui a presença de febre, mialgia ou fadiga, tosse produtiva e/ou tosse não produtiva dispneia, fadiga ou mialgias na maioria dos casos. Existem ainda relatados de sintomas como a cefaleia, dor de garganta, hemoptises e sintomas gastrointestinais como a diarreia, náusea e dor abdominal, mas com menor incidência ${ }^{(3-6)}$.

Neste contexto da pandemia COVID-19 a maioria dos doentes infetados com SARS-CoV-2 apresenta doença ligeira (cerca de $80 \%$ ), com prognóstico favorável( ${ }^{(7)}$.
Doentes com sintomatologia severa, podem desenvolver dispneia e hipoxemia, no período de 1 semana de infeção, que pode progredir rapidamente para Síndrome de Dificuldade Respiratória Aguda no Adulto/ acute respiratory distress syndrome (SDRA/ARDS) ou falência orgânica(3). Estima-se que entre 6 a $10 \%$ dos doentes infetados tenham necessidade de hospitalização, com necessidade de internamento em Unidades de Cuidados Intensivos ${ }^{(7)}$, em que os doentes mais idosos ou doentes com doenças crónicas (como hipertensão, diabetes mellitus, etc), podem ter um prognóstico mais reservado ${ }^{(3)}$.

A ARDS foi inicialmente definida em 1967, sendo que a sintomatologia associada inclui hipoxemia, edema pulmonar não cardiogénico, redução da compliance pulmonar, aumento do trabalho respiratório e a necessidade de ventilação com pressão positiva, em situações como trauma, pneumonia, sépsis e aspiração (p.e. conteúdo gástrico) ${ }^{(8)}$.

Uma das intervenções propostas para coadjuvar o tratamento em pessoas com ARDS em contexto de COVID é o decúbito ventral ou Prone Position ${ }^{(3)}$.

Os primeiros estudos sobre decúbito ventral, não demonstraram qualquer impacto na mortalidade, apesar de terem sido realizados numa fase da ventilação protetora, em que os doentes que se encontravam nesta posição apresentavam ARDS ligeira e permaneciam nela por curtos períodos. Posteriormente, com o desenvolvimento da ventilação protetora verificou-se que a mortalidade, nos doentes com ARDS severo em decúbito ventral, diminui significativamente quando comparada com a posição supina ou decúbito dorsal( ${ }^{(9)}$. Assim, uma meta-analise conclui que um doente com ARDS moderado a severo, conectado a ventilação mecânica em decúbito ventral, no máximo até 16 horas, apresentaram uma diminuição da mortalidade ${ }^{(10)}$.

A posição de decúbito ventral torna a ventilação mais homogénea, diminuindo a distensão alveolar ventral e o colapso alveolar dorsal. Neste contexto, existe uma redução da compressão pulmonar, levando a um aumento da perfusão(11). Seguidamente é utilizada maioritariamente em doentes com ARDS moderado a severo em que a relação pressão parcial de oxigénio $\left(\mathrm{PaO}_{2}\right)$ / fração de oxigénio $\mathrm{FiO}_{2}$ se encontra abaixo de $150 \mathrm{mmHg}$ e necessitam de um $\mathrm{FiO}_{2}$ acima de 0.6 $(60 \%)^{(12)}$. 
Podemos afirmar que a ARDS é uma consequência da SARS-Cov-2, sendo estimado que dos doentes infetados, $17 \%$ possa a vir desenvolver $\operatorname{ARDS}^{(13)}$. Assim, visto que 0 decúbito ventral é um tratamento não invasivo da ARDS, doentes infetados com SARS-Cov-2 podem também vir a beneficiar deste posicionamento.

Por fim, esta intervenção também veio apresentar contraindicações como em doentes com instabilidade da coluna, doentes que sofreram um trauma ou foram submetidos a uma cirurgia cardíaca no período de 24 horas e também doentes sob técnica de Extra Corporeal Membrane Oxygenation (ECMO) veno-arterial (12).

O objetivo desta revisão foi identificar os efeitos da posição ventral (prone position) no tratamento de doentes com ARDS causada por SARS-Cov- 2 .

\section{MATERIAL E MÉTODOS}

\section{Aspetos éticos}

Por se tratar de um estudo de revisão, não houve necessidade de submeter o protocolo da pesquisa a uma comissão de ética. No entanto, em toda revisão foram respeitadas as ideias, conceitos e definições dos autores dos artigos que compuseram este estudo.

\section{Tipo de estudo}

Este estudo apresentam o desenho de uma Revisão Integrativa da Literatura (RIL)(14-15). É um método que permite a síntese de conhecimento e a incorporação dos resultados de estudos significativos na prática clínica. Este tipo de revisão tem sido apontado como uma ferramenta importante na síntese da evidência disponíveis sobre determinada temática e direciona a prática fundamentada em conhecimento científico, ou seja, para a prática baseada na evidência ${ }^{(15)}$.

\section{Colheita de dados}

Neste estudo seguiram-se as seis etapas de RIL: identificação do tema e elaboração da questão de pesquisa, amostragem ou pesquisa da literatura, colheita de dados, análise crítica dos estudos incluídos, interpretação e discussão dos resultados e apresentação da revisão/ síntese de conhecimento ${ }^{(15)}$.

Tendo em consideração objetivo desta RIL, a formulação a seguinte questão norteadora com base no acrónimo $\mathrm{PICo}^{(16)}$ : Quais os efeitos da posição de ventral/prono position (I - Interest phenomenon ) em doentes com ARDS ( $P$ - Patient) causada por SARS-Cov2 (Co - Context).? O levantamento das produções científicas foi efetuado em maio de 2020 nas seguintes bases de dados eletrónicas: Medical Literature Analysis and Retrieval System Online (MEDLINE), SCOPUS e complementada no google académico.

0 acesso às bases de dados eletrónicas selecionadas foi realizado através do Portal Regional da Biblioteca Virtual em Saúde (BVS) e SCOPUS. Foi utilizada a equação booleana (Prone Position) AND ((COVID-19) OR (SARS-Cov-2)), no campo destinado à pesquisa em
"Título, resumo, assunto". A Figura 1 mostra o fluxograma das etapas seguidas até a obtenção da amostra final.

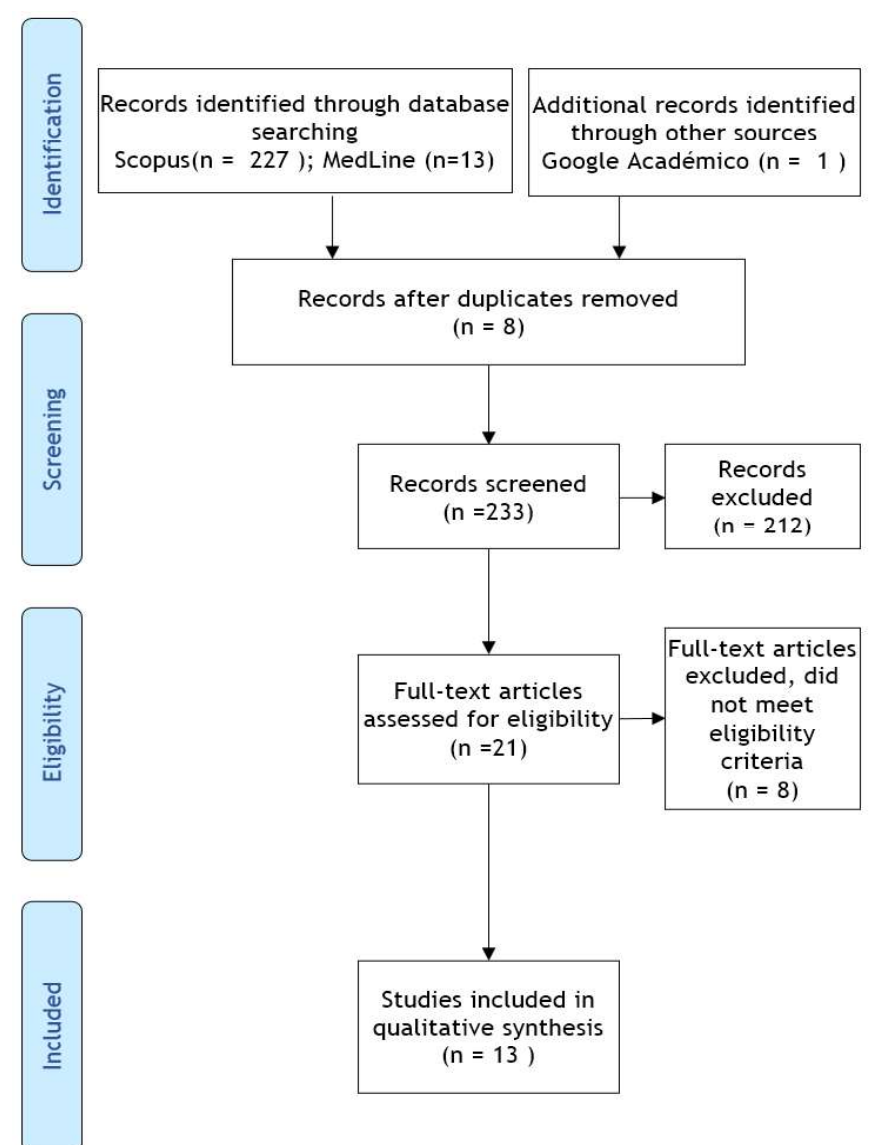

Figura 1 - Fluxograma PRISMA, identificação, triagem, elegibilidade e inclusão

Os critérios de inclusão adotados para a amostra deste estudo foram os seguintes: estudos do tipo relato de experiência, estudo de caso e artigos originais, veiculados em periódicos nacionais e internacionais; que tenham utilizado abordagens qualitativas, quantitativas e quanti-qualitativa; publicados entre janeiro de 2019 e maio de 2020, nos idiomas português, espanhol e inglês; disponíveis online e com texto na íntegra. Os critérios de exclusão são: estudos que trataram da posição de ventral (Prone position) em outras situações de ARDS que não por SARS-Cov-2 ou que não respondessem à questão norteadora.

\section{Extração e Análise dos dados}

Após a aplicação dos critérios de inclusão e exclusão restaram 13 artigos que constituíram a amostra final desta RIL, conforme demonstrado na tabela 1, destacando o título, país, o ano de publicação e os principais resultados de cada artigo selecionado. Nesta etapa, os dados foram avaliados, com o objetivo de constatar sua adequação ao tema da pesquisa. Foi realizada uma triagem por meio da leitura crítica dos estudos selecionados, os quais responderam à questão norteadora desta RIL. Utilizou-se um instrumento de coleta de dados, previamente elaborado para este fim, contendo dados de identificação (autores, ano de publicação e país), revista, objetivos, resultados/ conclusões e desenho/nível de evidência. Os níveis de 
evidência (NE) dos estudos foram classificados de acordo com critérios da Registered Nurses Association of Ontario: NE III - estudos descritivos e estudos de coorte, series de caso; NE IV - artigos de opinião de peritos, revisões narrativas ${ }^{(17)}$.

\section{RESULTADOS}

Foram identificados 241 artigos com a equação booleana nas bases de dados SCOPUS, MedLine e Google académico, após leitura do título, resumo e aplicação dos critérios de elegibilidade foram incluídos $13^{(18-30)}$ artigos (figura 1).

Todos os artigos foram publicados em 2020, são oriundos da Alemanha ${ }^{(19)}$, Brasil(28), Canadá(29), China ${ }^{(25)}$, Espanha $^{(18)}$, Estados Unidades da América ${ }^{(20,23)}$, França ${ }^{(22,30)}$, Irão ${ }^{(26)}$ e Itália( ${ }^{(21,24,27)}$. As amostras variam entre 1 caso $^{(23,30)}$ e $62^{(27)}$ doentes com COVID-19 com ARDS moderada submetidos a Ventilação não invasiva (VNI). O nível de evidência varia entre III ${ }^{(18,20-24,26-28,30)}$ e IV $^{(19,25,29)}$.

Os dados foram sintetizados em 3 grandes temas, indicações, frequência da alternância de decúbito e duração e por últimos efeitos do decúbito ventral.
As indicações do decúbito ventral por SARS-CoV-2 moderada a grave, em contexto de unidade de cuidados intensivos $(\mathrm{UCl})^{(18-19,21-22,25)}$, acordados e não ventilados $^{(20)}$, em enfermaria para doentes COVID 19 (2628) com oxigénio por cânula nasal(23), ventilação não invasiva (VNI) com capacete ${ }^{(24)}$, grávida de 25 semanas em $\mathrm{UCl}^{(30)}$.

A alternância de decúbitos entre decúbito ventral e lateralizações deve ser efetuada de 2 em 2 horas $^{(30)}, 12$ a 16 horas por dia nas situações de ARDS grave a moderado $^{(19)}$.

Os principais benefícios identificados foram: melhoria dos parâmetros ventilatórios nomeadamente, diminuição da sensação de dispneia(26), frequência respiratória menor (27), melhoria da mecânica ventilatória e trocas gasosas ${ }^{(25)}$, melhoria das saturações de Oxigénio(20-21,23,27-30) e valores de gasometria ${ }^{(22,29-30)}$, relação $\mathrm{PaO}_{2} / \mathrm{FiO}_{2}{ }^{(21)}$, melhoria do fluxo para as áreas de ventilação/perfusão(24), evitouse intubação para ventilação mecânica invasiva ${ }^{(18)}$, alta precoce $^{(18,23-24)}$, diminuição da média de internamento $^{(26)}$, diminuição da mortalidade ${ }^{(21,25-26)}$ aos

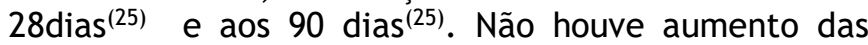
úlceras por pressão (UPP) ${ }^{(22)}$.

Tabela 1: descrição dos artigos incluídos.

\begin{tabular}{|c|c|c|c|}
\hline $\begin{array}{c}\text { Autor/ } \\
\text { país /ano }\end{array}$ & Revista & Objetivo & Resultados/conclusões \\
\hline
\end{tabular}

\begin{tabular}{|c|c|c|}
\hline $\begin{array}{l}\text { Barrasa et } \\
\text { al., 2020, } \\
\text { Espanha(18 } \\
\text { ) }\end{array}$ & $\begin{array}{l}\text { Anaesthesia } \\
\text { Critical Care } \\
\text { \& Pain } \\
\text { Medicine }\end{array}$ & $\begin{array}{l}\text { Relatar a epidemiologia } \\
\text { dos primeiros doentes } \\
\text { admitidos com SARS-CoV-2 } \\
\text { em Unidades de cuidados } \\
\text { intensivos (UCI) } \\
\text { espanholas, vinte dias } \\
\text { após o primeiro } \\
\text { internamento num hospital } \\
\text { de Espanha. }\end{array}$ \\
\hline $\begin{array}{l}\text { Bein et al., } \\
\text { 2020; } \\
\text { Alemanha( } \\
\text { 19) }\end{array}$ & $\begin{array}{l}\text { Anästhesiol } \\
\text { Intensivmed } \\
\text { Notfallmed } \\
\text { Schmerzther }\end{array}$ & $\begin{array}{l}\text { Descrever o conhecimento } \\
\text { sobre o disgnóstico e } \\
\text { tratamento do COVID } 19\end{array}$ \\
\hline $\begin{array}{l}\text { Caputo et } \\
\text { al, 2020, } \\
\text { Estados } \\
\text { Unidos } \\
\text { América(2 } \\
\text { 0) }\end{array}$ & $\begin{array}{l}\text { Academic } \\
\text { Emergency } \\
\text { Medicine }\end{array}$ & $\begin{array}{l}\text { Descrever o uso do } \\
\text { decúbito ventral precoce } \\
\text { em doentes COVID-19 } \\
\text { acordados e não } \\
\text { intubados. }\end{array}$ \\
\hline
\end{tabular}

0)

Relatar a epidemiologia

Incluídos 48 doentes. No início desta doença, doentes não
intubados podem se beneficiar do decúbito ventral antes da intubação. Três dos doentes foram tratados dessa maneira sem intubação e tiveram alta precoce. 0 benefício do decúbito ventral em doentes intubados e a ventilação protetora deve ser restrita àqueles que desenvolvem lesão pulmonar aguda.

Evidência

Foram incluídos 50 doentes. Após 5 minutos em decúbito
Estudo prospetivo NE: III.
O decúbito ventral está recomendado no doente COVID-19 com ARDS moderada a grave, 12-16 horas por dia ventral as saturações melhoraram 94\% (amplitude interquartil entre 90 a 95).

A auto-pronação precoce no departamento de emergência demonstrou uma melhor saturação de oxigênio nos doentes positivos para COVID-19.

Estudo com 10 doentes. A ventilação em decúbito ventral é um método estabelecido para melhorar a oxigenação na síndrome do desconforto respiratório agudo grave (ARDS), e sua aplicação pode reduzir a taxa de mortalidade. A oxigenação melhorou significativamente durante a ventilação em decúbito ventral. Curiosamente, a $\mathrm{PaO} 2$ / FiO2 registada na posição supina após um teste de pronação prolongado foi significativamente maior do que a $\mathrm{PaO} 2$ / FiO2 medida antes da pronação. Não houve aumento de incidência de úlcera por pressão.
Carsetti et al., 2020, Itália(21)
O objetivo deste relatório foi avaliar a viabilidade e Critical Care eficácia da ventilação em decúbito ventral além das $16 \mathrm{~h}$ usuais.
Revisão narrativa NE: IV

Estudo piloto NE: III

Estudo retrospetivo NE: III 


\begin{tabular}{l|l|l}
\hline & & $\begin{array}{l}\text { Avaliar o efeito do } \\
\text { decúbito ventral em } \\
\text { doentes não intubados }\end{array}$ \\
$\begin{array}{l}\text { Elharrar et } \\
\text { al. 2020, } \\
\text { França(22) }\end{array}$ & JAMA & $\begin{array}{l}\text { com COVID-19 e com } \\
\text { insuficiência respiratória } \\
\text { aguda hipoxémica }\end{array}$ \\
\hline
\end{tabular}

\begin{tabular}{l|l|l} 
Elkattawy, & Respiratory & Verificar o efeito do \\
Noori; & Medicine & decúbito ventral num caso \\
2020, & Case Reports & de coronavírus \\
EUA(23) & de
\end{tabular}

$\operatorname{EUA}(23)$

Medicine

Case Reports
Em 24 doentes com insuficiência respiratória aguda hipoxémica o uso do decúbito ventral não alterou o suprimento de oxigênio ou fração de oxigênio (FIO2). Quatro doentes não toleraram o decúbito ventral por mais de uma hora (exigindo intubação posterior);

6 de 15 doentes que toleraram o decúbito ventral mostraram aumento média da $\mathrm{PaO} 2$ superior a $20 \%$ em relação à linha de base.
Estudo serie de casos,

Com avaliação Antes e depois.

NE: III

Relato de caso. NE: III entanto, dentro de algumas horas em pronação, ele apresentou alívio sintomático significativo com a melhoria da oxigenação. Tendo alta após um dia de pronação.

Referem que os doentes com COVID-19 podem beneficiar da combinação de hCPAP precoce em níveis moderados de PEEP (ou seja, $10 \mathrm{cmH} 20$ ) e decúbito ventral, para evitar sobredistensão das áreas saudáveis do pulmão, retardando a progressão da doença e permitindo que ganhem tempo na recuperação. O hCPAP provavelmente será eficaz, mantendo o pulmão aberto e reduzindo a mistura venosa, desviando o fluxo para melhores áreas de ventilação/perfusão

Ventilação em decúbito ventral melhora a mecânica pulmonar e troca de gasosas sendo atualmente é recomendado pelas diretrizes. Este posicionamento, quando planeado, não deve ser uma tentativa final desesperada, mas deve ser considerada no início estágios da doença, pois as evidências sugerem que a aplicação precoce da ventilação prolongada em decúbito ventral diminui a mortalidade aos 28 e aos 90 dias nos doentes com ARDS grave. Atualmente, a ventilação em decúbito ventral é amplamente utilizada em doentes críticos em Wuhan.

Aplicação de decúbito ventral em 10 doentes selecionados aleatoriamente, que tinham COVID-19 (70\% do sexo masculino e $30 \%$ do sexo feminino) e foram internados numa enfermaria específica para doentes COVID-19. A sensação de dispneia diminuiu em $40 \%$ dos casos e todos doentes tiveram alta do hospital. 0 tempo médio de internamento foi de 4,8 dias e não houve óbitos.

A utilização de VNI em decúbito ventral em 62 doentes com COVID-19 e ARDS nas enfermarias gerais de um hospital na Itália foi viável. A frequência respiratória foi menor e a oxigenação foi maior durante e após o decúbito ventral do que no início. Ainda não foi determinado se a intubação foi evitada ou atrasada.

Opinião de peritos. NE: III

Relato de

\begin{tabular}{|c|c|c|}
\hline $\begin{array}{l}\text { Moghadam } \\
\text { et al, } \\
\text { 2020, } \\
\text { Irão(26) }\end{array}$ & $\begin{array}{l}\text { Brazilian } \\
\text { Journal of } \\
\text { Anesthesiolo } \\
\text { gy }\end{array}$ & $\begin{array}{l}\text { Relatar a experiência de } \\
10 \text { casos submetidos a } \\
\text { decúbito ventral }\end{array}$ \\
\hline
\end{tabular}

Sartini et al., 2020, itália(27)

JAMA doentes submetidos à VNI em decúbito ventral fora da $\mathrm{UCl}$, independentemente do dia em que começaram a usar essa técnica.

\begin{tabular}{l|l|l} 
Sztajnbok & Respiratory & Relatar de dois caso \\
et al, & Medicine & doentes com pneumonia \\
2020, & Case Reports & por COVID-19 \\
Brasil(28) & &
\end{tabular}

Telias et

al., 2020,

Canadá(29)

JAMA

Discutir a utilidade do decúbito ventral durante a respiração espontânea em doentes com COVID-19

O decúbito ventral é uma estratégia bem conhecida de suporte ventilatório para melhorar os níveis de oxigenação. 0 autoposicionamento em ventral pode ser usado no tratamento de doentes com pneumonia por COVID-19

0 decúbito ventral durante a respiração espontânea e assistida em doentes com insuficiência respiratória hipoxémica aguda pode tornar-se uma intervenção terapêutica num futuro próximo. A tolerância é uma limitação da técnica, os efeitos fisiológicos não são esclarecidos e os benefícios de sessões muito curtas podem ser questionáveis. A melhoria da oxigenação durante o decúbito ventral pode impedir que os médicos tomem decisões sobre a intubação apenas com base na hipoxemia.

0 doente foi lateralizado por períodos de 2 horas durante o dia. 0 estado respiratório da doente melhorou com decúbito ventral e lateralização. A SatO2 aumentou enquanto os parâmetros do oxigênio de alto fluxo permaneceram estáveis e também houve melhoria dos parâmetros gasimétricos. No $16^{\circ}$ dia na $\mathrm{UCl}$, foi transferida para o

departamento de obstetrícia e recebeu alta 8 dias depois.
al, 2020,
França(30)

Relatar o caso de uma mulher de 21 anos, gravida de 25 semanas

and $\quad$ mulher de 21 a

Relato de experiência (opinião de peritos) NE: IV casos. NE: III

\section{Estudo} descritivo transversal Série de casos NE: III

Relato de caso. NE: III

Opinião de peritos NE: IV

Relato de caso. NE: III 


\section{DISCUSSÃO}

O tempo de permanência dos doentes em cuidados intensivos, devido a SARS-CoV-2 parece ser relativamente longo, implicando a imobilização no leito e muitas horas decúbito ventral. ${ }^{(31)}$. 0 recurso a este posicionamento é também defendido em ambiente de enfermaria pela Sociedade de Cuidados Intensivos do Reino Unido defende o uso de decúbito ventral em doentes conscientes na enfermaria que requerem $\geq 28 \%$ de oxigênio (32).

A utilização do decúbito ventral tem benefícios em doentes em enfermaria, através do autoposicionamento ${ }^{(28)}$, associado a oxigénio por cânula nasal(23), oxigênio de alto fluxo ${ }^{(30)}$, VNI com capacete ${ }^{(24)}$ e ventilação mecânica invasiva $(\mathrm{VMI})^{(18-19,21-22,25)}$. Está indicada nas situações de pneumonia associada a SARSCoV-2 moderada a grave em contexto de UCI (18-19,21. 22,25,30), na enfermaria especifica para COVID 19 ${ }^{(26-28)}$. A sua utilização em contexto de enfermaria e nos serviços de urgência/emergência pode evitar intubações invasivas $^{(18)}$ e favorecer a alta precoce ${ }^{(18,23-24)}$. Como foi referido anteriormente existem benefícios documentados da combinação do decúbito ventral associados a outras intervenções terapêuticas como um suplemente de oxigénio por cânula nasal, oxigénio de alto fluxo, VNI com capacete e VMI, pois permite o recrutamento alveolar, melhorando a mecânica ventilatória e trocas gasosas ${ }^{(25)}$, promovendo o desvio do fluxo para as áreas de melhor ventilação/perfusão(25), com consequente melhoria das saturações periféricas ${ }^{(20-21,23,27-30)}$ e valores gasimétricos ${ }^{(22,29-30)}$,

0 fato da intervenção em decúbito ventral ser recomendada entre 12 a 16 horas ${ }^{(19)}$, com alternâncias para lateralização de 2 em 2 horas ${ }^{(30)}$, implica uma sobrecarga nos recursos humanos envolvidos nos cuidados a estes doentes. A mobilização e posicionamento do doente sob VMI em decúbito ventral deve ser realizado por profissionais de saúde (4-5 elementos) devidamente treinados ${ }^{(33)}$ que devem ter atenção da possível desconexão do tubo orotraqueal do ventilador $^{(11)}$.

Apesar dos benefícios, existem estudos que referem o aumento do risco de desenvolvimento de UPP e a obstrução do tubo orotraqueal com a utilização do decúbito ventral ${ }^{(11)}$. Contudo, num estudo incluído refere que não houve aumento da incidência do UPP(22).

Outra complicação que pode surgir com o decúbito ventral, tanto em doentes conscientes quanto nos submetidos à VMI, é a ocorrência de paragem cardíaca na posição ${ }^{(32)}$, neste sentido tem de haver uma permanente acompanhamento e monitorização para detetar precocemente mudanças significativas no estado do doente e consequentes complicações.

Implicações para a prática de enfermagem e políticas de saúde

Os profissionais de saúde mais habilitados para realizar estas intervenções, são os enfermeiros e os enfermeiros de reabilitação, uma vez que possuem conhecimento científico e clínico essencial à redução do risco de complicações ${ }^{(12)}$. Por conseguinte, para garantir a segurança do doente e do profissional é recomendado o conhecimento e treino da técnica, assim como, o uso adequado dos equipamentos de proteção individual ${ }^{(11)}$. Além disso, o conhecimento das complicações e a sua prevenção são a chave para a segurança do doente quando posicionado em decúbito ventral ${ }^{(\mathfrak{1 2})}$.

\section{Limitações do estudo}

Esta pesquisa foi efetuada recorrendo a base de dados com grande fator de impacto, no entanto, foram utilizados apenas estudos em inglês e de evidência III e IV. Recomendam-se outros estudos, que envolvam outras línguas para ter uma maior abrangência do conhecimento. Recomenda-se a realização de estudos primários com um desenho experimental ou quasiexperimental, com amostra mais robustas para aumentar o NE.

\section{CONSIDERAÇÕES FINAIS}

Com estas pesquisa alcançou-se o objetivo previamente definido, foi possível identificar as indicações, cuidados e efeitos do decúbito ventral como coadjuvante ao tratamento da SARS-CoV-2. Além de melhorar os parâmetros ventilatórios e gasimétricos, melhora os sintomas de dispneia, facilita a recuperação, com consequente, alta precoce, diminuição dos dias de internamento e redução da mortalidade aos 28 e 90 dias.

Os enfermeiros em geral e os enfermeiros especialistas em enfermagem de reabilitação em particular, neste contexto devem ser possuidores de conhecimento científico baseado nas últimas evidencias e devem ser treinados, com recurso a simulação virtual e/ou física, não só na realização da técnica correta como também na utilização dos materiais de proteção individual para que seja assegurada a segurança para o doente e da equipa de saúde envolvida no procedimento.

\section{REFERÊNCIAS BIBLIOGRÁFICAS}

1. Singhal T. A review of coronavirus disease-2019 (COVID-19). Indian J Pediatr. 2020; 87, 281-286. Disponível em: https://doi.org/10.1007/s12098-020-03263-6.

2. Rajagopal K, Keller SP, Akkanti B, Bime C, Loyalka P, et al. Advanced Pulmonary and Cardiac Support of COVID-19 Patients: Emerging Recommendations From ASAIO-a Living Working Document. Circ Heart Fail. 2020 May;13(5):e007175. Disponível em: https://doi.org/10.1161/CIRCHEARTFAILURE.120.007175.

3. Wu C, Chen X, Cai Y, Zhou X, Xu S, Huang Het al. Risk factors associated with acute respiratory distress syndrome and death in patients with coronavirus disease 2019 pneumonia in Wuhan, China. JAMA Intern Med. 2020 Mar 13. Disponível em: https://doi.org/10.1001/jamainternmed.2020.0994.

4. Jiang F, Deng L, Zhang L, Cai Y, Cheung CW, Xia Z. Review of the clinical characteristics of coronavírus disease 2019 (COVID-19). J Crit Care Med. 2020 Mar 4:1-5 Disponível em: https://doi.org/10.1007/s11606-020-05762-w.

5. Del Rio C, Malani PN. COVID-19-new insights on a rapidly changing epidemic. JAMA. 2020 Apr 14;323(14):1339-40. Disponível https://jamanetwork.com/journals/jama/articleabstract/2762510

6. Zhou F, Yu T, Du R, Fan G, Liu Y, Liu Z, Xiang J, Wang Y, Song B, 
Gu X, Guan L. Clinical course ans risk factors for mortality of adult inpatients with COVID-19 in 24 Wuhan, China: a retrospective cohort study. Lancet. 2020 Mar 11; 395(10229): 1054-1062 Disponível em: https://doi.org/10.1016/S0140-6736(20)30566-3

7. Wang $D$, Hu B, Hu C, Zhu F, Liu X, Zhang J, Wang B, Xiang $H$, Cheng Z, Xiong Y, Zhao Y. Clinical characteristics of 138 hospitalized patients with 2019 novel coronavirus-infected pneumonia in Wuhan, China. JAMA. 2020 Mar 17;323(11):1061-9. Disponível em: https://doi.org/10.1001/jama.2020.1585.

8. Matthay MA, Zemans RL, Zimmerman GA, Arabi YM, Beitler JR, Mercat A, Herridge M, Randolph AG, Calfee CS. Acute respiratory distress syndrome. Nat Rev Dis Primers. 2019 Mar 14;5(1):1-22. Disponível em: https://doi.org/10.1038/s41572-019-0069-0

9. Guérin C, Reignier J, Richard JC, Beuret P, Gacouin A, Boulain T, Mercier E, Badet M, Mercat A, Baudin O, Clavel M. Prone positioning in severe acute respiratory distress syndrome. $\mathrm{N}$ Engl J Med. 2013 Jun 6;368(23):2159-68. Disponível em: https://www.nejm.org/doi/pdf/10.1056/nejmoa1214103

10. Sud S, Friedrich JO, Adhikari NK, Taccone P, Mancebo J, Polli F, Latini R, Pesenti A, Curley MA, Fernandez R, Chan MC. Effect of prone positioning during mechanical ventilation on mortality among patients with acute respiratory distress syndrome: a systematic review and meta-analysis. CMAJ. 2014 Jul 8;186(10):E381-90. Disponível em. https://doi.org/10.1503/cmaj.140081.

11. Alhazzani W, Møller MH, Arabi YM, Loeb M, Gong MN, Fan E, Oczkowski S, Levy MM, Derde L, Dzierba A, Du B. Surviving Sepsis Campaign: guidelines on the management of critically ill adults with Coronavirus Disease 2019 (COVID-19). Crit Care Med. 2020 Mar 28:1-34.

12. Bamford P, Denmade C, Newmarch C. Guidance for prone positioning in adult critical care. Intensive Care Soc 2019; 1-39. Disponível em https://www.ics.ac.uk/ICS/News Statements/Prone Positioning in Adu It_Critical_Care.aspx

13. Chen N, Zhou M, Dong X, Qu J, Gong F, Han Y, Qiu Y, Wang J, Liu $Y$, Wei Y, Yu T. Epidemiological and clinical characteristics of 99 cases of 2019 novel coronavirus pneumonia in Wuhan, China: a descriptive study. Lancet. 2020 Feb 15;395(10223):507-13. Disponível em: https://doi.org/10.1016/S0140-6736(20)30211-7.

14. Sousa LM, Firmino CF, Marques-Vieira CM, Severino SS, Pestana HC. Revisões da literatura científica: tipos, métodos e aplicações em enfermagem. Rev Port Enferm Reabil [Internet]. 2018 Jun 22[citado 2020 Maio 21];1(1):45-54. Disponível em: http://dx.doi.org/10.33194/rper.2018.v1.n1.07.4391

15. Sousa LM, Marques-Vieira C, Severino SS, Antunes AV. Metodologia de Revisão Integrativa da Literatura em Enfermagem. Rev Inv Enferm [Internet]. 2017 [citado 2020 Maio 21]; S2 (21): 17-26. Disponível em: http://hdl.handle.net/20.500.12253/1311

16. Sousa LM, Marques JM, Firmino CF, Frade F, Valentim OS, Antunes AV. Modelos de formulação da questão de investigação na Prática Baseada na Evidência. Rev Inv Enferm [Internet]. 2018 [citado 2020 Maio 21]; S2 (23): 31-9. Disponível em: http://hdl.handle.net/20.500.12253/1287

17. Registered Nurses'Association of Ontario Falls prevention: building the foundations for the patient safety. A self-learning package. Toronto: Registered Nurses' Association of Ontario 2007.

18. Barrasa H, Rello J, Tejada S, Martín A, Balziskueta G, Vinuesa C, Fernández-Miret B, Villagra A, Vallejo A, San Sebastián A, Cabañes S. SARS-CoV-2 in Spanish Intensive Care: Early experience with 15-day survival in Vitoria. Anaesth Crit Care Pain Med. $2020 \quad$ Apr $9 . \quad$ Disponivel em: https://doi.org/10.1016/j.accpm.2020.04.001

19. Bein B, Bachmann M, Huggett $S$, Wegermann P. SARS CoV2/COVID-19: Evidence-Based Recommendation on Diagnosis and Therapy. Anasthesiologie, Intensivmedizin, Notfallmedizin, Schmerztherapie: AINS. 2020 Apr 9;55(4):257-65. Disponivel em: https://doi.org/10.1055/a-1156-3991

20. Caputo ND, Strayer RJ, Levitan R. Early Self-Proning in Awake, Non-intubated Patients in the Emergency Department: A Single ED's Experience during the COVID-19 Pandemic. Acad Emerg Med. 2020 May;27(5):375-8. Disponivel em: https://doi.org/10.1111/acem.13994

21. Carsetti A, Paciarini AD, Marini B, Pantanetti S, Adrario E, Donati A. Prolonged prone position ventilation for SARS-CoV-2 patients is feasible and effective. Crit Care. 2020 Dec;24(1):1-3. Disponivel em: https://doi.org/10.1186/s13054-020-02956-w

22. Elharrar $X$, Trigui $Y$, Dols $A-M$, et al. Use of prone positioning in nonintubated patients with COVID-19 and hypoxemic acute respiratory failure. JAMA. Published online May 15, 2020. Disponivel em: https://doi.org/10.1001/jama.2020.825

23. Elkattawy S, Noori M. A case of improved oxygenation in SARS CoV-2 positive patient on nasal cannula undergoing prone positioning. Respir Med Case Rep. 2020 May 4; 30:101070. Disponivel em: https://doi.org/10.1016/j.rmcr.2020.101070

24. Longhini F, Bruni A, Garofalo E, Navalesi P, Grasselli G, Cosentini R, Foti G, Mattei A, Ippolito MC, Accurso G, Vitale F. Helmet Continuous Positive Airway Pressure and prone positioning: a proposal for an early management of COVID-19 patients. Pulmonology. 2020 Apr $30 . \quad$ Disponivel em: https://static.elsevier.es/covid/PULMOE_1480.pdf

25. Meng L, Qiu H, Wan L, Ai Y, Xue Z, Guo Q, Deshpande R, Zhang L, Meng J, Tong C, Liu H. Intubation and Ventilation amid the COVID19 OutbreakWuhan's Experience. Anesthesiology: Anesthesiology. 2020 Mar 26;132: 1317-1332. Disponível em: https://doi.org/10.1097/ALN.0000000000003296

26. Moghadam VD, Shafiee H, Ghorbani M, Heidarifar R. Prone positioning in management of COVID-19 hospitalized patients. Braz J Anesthesiol. 2020 May 16. Disponivel em: https://doi.org/10.1016/j.bjane.2020.05.001

27. Sartini C, Tresoldi M, Scarpellini P, Tettamanti A, Carcò F, Landoni G, Zangrillo A. Respiratory Parameters in Patients With COVID-19 After Using Noninvasive Ventilation in the Prone Position Outside the Intensive Care Unit. JAMA. 2020 May 15. Disponivel em: https://doi.org/10.1001/jama.2020.7861

28. Sztajnbok J, Maselli-Schoueri JH, de Resende Brasil LM, de Sousa LF, Cordeiro CM, Borges LM, Malaque C. Prone positioning to improve oxygenation and relieve respiratory symptoms in awake, spontaneously breathing non-intubated patients with COVID-19 pneumonia. Respir Med Case Rep. 2020 May 19, 30:101096. Disponivel em: https://doi.org/10.1016/j.rmcr.2020.101096

29. Telias I, Katira BH, Brochard L. Is the Prone Position Helpful During Spontaneous Breathing in Patients With COVID-19?. JAMA. 2020 May 15. Disponivel em: https://doi.org/10.1001/jama.2020.8539

30. Vibert F, Kretz M, Thuet V, Barthel F, De Marcillac F, Deruelle $P$, Lecointre L. Prone positioning and high-flow oxygen improved respiratory function in a 25 -week pregnant woman with COVID19. Eur J Obstet Gynecol Reprod Biol. 2020 May 13. . Disponivel em: https://doi.org/10.1016/j.ejogrb.2020.05.022.

31. Kiekens C, Boldrini P, Andreoli A, Avesani R, Gamna F, Grandi M, Lombardi F, Lusuardi M, Molteni F, Perboni A, Negrini S. Rehabilitation and respiratory management in the acute and early post-acute phase." Instant paper from the field" on rehabilitation answers to the Covid-19 emergency. Eur J Phys Rehabil Med. 2020 Apr 15:06305-4.

32. Barker J, Koeckerling D, West R. A need for prone position CPR guidance for intubated and non-intubated patients during the COVID-19 pandemic. Resusc 2020 May 1; 151:135-136. Disponivel em: OI: https://doi.org/10.1016/j.resuscitation.2020.04.029

33. Schub ERB, Pilgrim JRBM. Patient Positioning (Critical Care Patients): Prone. Pravikoff D RPF, editor. CINAHL Nursing Guide [Internet]. 2020 May 1 [cited 2020 May 23]; Available from: http://search.ebscohost.com/login.aspx?direct=true\&site=edslive\&db=nup\&AN=T705572 